\title{
Axitinib: The evidence of its potential in the treatment of advanced thyroid cancer
}

This article was published in the following Dove Press journal:

Core Evidence

8 June 2009

Number of times this article has been viewed

\author{
Hari A Deshpande' \\ Scott Gettinger' \\ Julie Ann Sosa ${ }^{2}$ \\ 'Yale Cancer Center, Department \\ of Medical Oncology, ${ }^{2}$ Division of \\ Endocrine Surgery, Department \\ of Surgery, Yale University School \\ of Medicine, New Haven, CT, USA
}

Correspondence: Hari A Deshpande Yale Cancer Center, 333 Cedar Street, FMP I24, New Haven, CT 06520, USA $\mathrm{Tel}+\mid 2037854$ |9|

Fax + I 2037853788

Email hari.deshpande@yale.edu
Introduction: Thyroid cancer is a rare disease with an incidence of around 37,000 cases per year. However, its incidence is rising faster than many other cancers and for men this disease ranks highest overall in the rate of increase (2.4\% annual increase) in cancer deaths. As the number of radioactive iodine-resistant thyroid cancers increases, the need for newer treatments has become more important. Axitinib is one of many new small molecule inhibitors of growth factor receptors that have shown promise in the treatment of many cancers. It targets the vascular endothelial growth factor receptors 1,2 and 3 .

Aims: The goal of this article is to review the published evidence for the use of axitinib in the treatment of thyroid cancer and define its therapeutic potential.

Evidence review: The major evidence of axitinib activity has appeared in meeting report abstracts. One phase II study has been published. This included patients with any histological type of thyroid cancer that was not amenable to treatment with radioactive iodine.

Clinical potential: To date, in phase II clinical studies axitinib has demonstrated antitumor activity in advanced refractory thyroid cancer. As a monotherapy it resulted in a $30 \%$ response rate with another $38 \%$ of patients having stable disease. Axitinib appears to have a good tolerability profile, with hypertension being the most common grade 3 or greater side effect.

Keywords: axitinib, thyroid cancer, vascular endothelial growth factor receptor

\section{Core evidence proof of concept summary for axitinib in thyroid cancer}

\begin{tabular}{|ll|}
\hline Outcome measure & Emerging evidence \\
\hline Efficacy & $\begin{array}{l}\text { Potential to use as monotherapy at a dose of } 5 \mathrm{mg} \\
\text { twice daily; stable disease achieved in } 38 \% \text { of patients } \\
\text { and progression-free survival of } 18.1 \text { months }\end{array}$ \\
Response rates & Evidence of activity with overall response rates of $30 \%$ \\
Biomarker expression & Decreases levels of soluble VEGFR 2 and 3. Blood \\
& pressure elevation may correlate with response \\
Tolerability & Well tolerated, with hypertension, fatigue, and \\
& proteinuria as the most common grade 3 side effects \\
\hline Abbreviations:VEGFR, vascular endothelial growth factor receptor.
\end{tabular}

\section{Scope, aims, and objectives}

Thyroid cancer is a relatively rare disease with an incidence of 37,340 cases per year. It retains an excellent prognosis with a mortality of only 1,590 per year. ${ }^{1}$ However, its incidence is rising and for men this disease ranks highest overall in the rate of increase (2.4\% annual increase) in cancer deaths. ${ }^{2}$ Radioactive iodine continues to be the initial treatment for differentiated thyroid cancer with distant metastatic disease 
and can produce five-year overall survival rates of around $50 \%$. The more aggressive variants as well as anaplastic and medullary subtypes of thyroid cancer however need better treatments to improve survival, and many agents have now shown some promise in clinical trials. ${ }^{3}$

Axitinib is an oral, potent, and selective inhibitor of vascular endothelial growth factor receptors (VEGFR) 1, 2, and 3. Axitinib was more than 10-fold less potent in inhibiting platelet-derived growth factor receptor beta (PDGFR) and c-KIT in cell-based assays. ${ }^{4}$ Thyroid cancers have elevated levels of VEGF compared with normal controls. Axitinib is one of a number of inhibitors of the VEGF pathway being studied in the treatment of thyroid cancers. The objective of this review is to evaluate the potential role of axitinib in the treatment of advanced thyroid cancers. Current options for the treatment of thyroid cancer, and the molecular basis underlying the use of tyrosine kinase inhibitors, are also discussed.

\section{Methods}

The English language medical literature was searched for relevant articles related to the use of axitinib in advanced thyroid cancer. The search terms used were "Axitinib or AG-013736," "thyroid cancer" and the articles published covered a period between 1998 to September 2008. The following databases were used:

- PubMed (http://www.ncbi.nlm.nih.gov/entrez)

- National Cancer Institute (http://www.cancer.gov/)

- Clinical Evidence (BMJ) (http://www.clinicalevidence. $\operatorname{com} /$ )

- Clinical Trials (http://www.clinicaltrials.com/)

The annual scientific sessions from the American Society of Clinical Oncology (ASCO), European Society of Medical Oncology (ESMO), American Association of Cancer Research (AACR), and American Thyroid Association (ATA; Thyroid) were searched for relevant meeting abstracts. A total of 27 articles were identified from those represented by systematic review. Articles were excluded because they were considered not relevant (Table 1). Two papers were identified ${ }^{5,6}$ replacing or supplementing earlier abstracts.

Few studies were identified for axitinib use specifically in thyroid cancer, but many references investigated its use and safety in the treatment of other malignancies with and without chemotherapy.

No health economics publications were found. One quality of life publication was found although it was for a different disease group.
Table I Evidence base included in the review

\begin{tabular}{lll}
\hline Category & \multicolumn{2}{l}{ Number of records } \\
\cline { 2 - 3 } & Full papers & Abstracts \\
\hline Initial search & 17 & 10 \\
Records included & 15 & 7 \\
Records excluded & 2 & 3 \\
Additional studies identified & 2 & 2 \\
Level I clinical evidence & 0 & 0 \\
Level 2 clinical evidence & 1 & 0 \\
Level >3 clinical evidence & 14 & 7 \\
Trials other than RCT & 14 & 7 \\
Case reports & 0 & 0 \\
Economic evidence & 1 & 0
\end{tabular}

Notes: For definition of levels of evidence, see Core Evidence website (http://www. dovepress.com/core-evidence-journal).

Abbreviation: RCT, randomized controlled trial.

\section{Disease overview}

Thyroid cancer is rare compared with other solid malignancies. It is the sixth most common cancer in women with an incidence of about 28,000 cases per year, but in men affects less than 10,000 individuals per year in the United States. ${ }^{1}$ Although relatively rare, the incidence of thyroid cancer more than doubled between 1984 and 2004 in the United States. Between 1995 and 2004, thyroid cancer was the third fastest growing cancer diagnosis, behind only peritoneum, omentum, and mesentery cancers and "other" digestive cancers. Similarly dramatic increases in thyroid cancer incidence have also been observed in Canada, Australia, Israel, and several European countries. ${ }^{7}$ The factors underlying this epidemic are not well understood, but may be partly a result of better screening and diagnosis with fine needle aspirations. ${ }^{8}$

Thyroid cancers arise from two cell types. The follicular cells give rise to the differentiated and undifferentiated types. Differentiated thyroid cancers account for $85 \%-95 \%$ of cases and consist mainly of papillary and follicular subtypes. The poorly and undifferentiated category includes anaplastic thyroid carcinoma, paradoxically one of the most aggressive and chemotherapy-resistant tumors known in humans. ${ }^{9}$ Parafollicullar or $\mathrm{C}$ cells are the cell of origin for medullary thyroid carcinoma, which may be familial or sporadic.

The epidemiology of thyroid cancer is unclear in the majority of cases but environmental and genetic factors are thought to contribute to an increased risk; these include environmental goiter, radiation exposure, and, in the case of medullary thyroid cancer, autosomal dominant inheritance. ${ }^{8}$

Differentiated thyroid cancers have an excellent prognosis when treated at an early stage and, even in cases 
of metastatic disease, patients can live for many years. The excellent survival of patients with thyroid cancer as well as the relatively low incidence of the disease has meant that it is probably not a significant economic burden on health care systems, although no published data could be found to confirm this. However, the incidence of the disease as mentioned is rising faster than almost any other cancer and therefore, if more aggressive variants continue to emerge, the toll on society is also likely to increase.

\section{Current therapy options}

Surgery plus radioactive iodine therapy has remained the standard treatment for early and locally advanced differentiated thyroid cancer. Several groups have demonstrated a survival advantage in patients treated with a total or near-total thyroidectomy followed by radioactive iodine treatment. The question of neck dissection is not as clear and probably should be reserved for patients with clinically positive nodes. ${ }^{10}$ Advanced metastatic thyroid cancers that are refractory to radioactive iodine have traditionally been treated with doxorubicin, platinum agents, taxanes, or combinations of the above based on little data. ${ }^{11}$ Cancers that are refractory to radioiodine have a poor prognosis with a 10-year survival rate of around $15 \% .{ }^{12}$ Currently doxorubicin is the only systemic agent approved for the treatment of metastatic thyroid cancer. In a randomized trial comparing doxorubicin $60 \mathrm{mg} / \mathrm{m}^{2}$ with the same dose of doxorubicin plus cisplatin $40 \mathrm{mg} / \mathrm{m}^{2}$ administered every three weeks a complete response was achieved in five of 43 patients. There was no difference in survival between the arms, but of the patients who had a complete response, two had a long-term survival. ${ }^{13}$ This impressive response rate however, was unable to be replicated in future studies and it soon became clear that new approaches to treatment were needed.

As mentioned earlier, thyroid cancers have elevated levels of VEGF compared with normal controls, and this pathway has become a target for some of the new small molecule inhibitors. VEGF proteins are important mediators of angiogenesis and it was felt that by inhibiting this pathway either with antibodies, inhibitors of the receptor tyrosine kinase or downstream proteins, it would be possible to treat resistant thyroid cancers. The recent explosion of multitargeted inhibitors in medical oncology has resulted in many of them being tested in refractory thyroid cancer. Motesanib (AMG 706) is a novel oral inhibitor of VEGF receptors, platelet-derived growth factor receptor, and KIT. It was tested in a phase II study in 93 patients who had progressive, radioiodine refractory, differentiated thyroid cancer. The authors noted a $14 \%$ response rate with $67 \%$ of patients achieving stable disease (35\% for 24 weeks or longer). ${ }^{14}$ The major side effects appeared to be hypertension and fatigue. Although this agent is not yet approved for treatment, it also represents a proof of concept and should lead to its use in confirmatory clinical trials.

The B-type Raf kinase (BRAF) in the mitogen-activated protein signaling pathway plays a key role in thyroid cancer. One common mutation occurs in about $29 \%-66 \%$ of papillary thyroid cancers. ${ }^{15}$ Sorafenib is a multi-tyrosine kinase inhibitor whose targets include BRAF and VEGFR 1 and 2 and has been studied in patients with advanced papillary thyroid cancer. Sorafenib activity was studied using immunohistochemistry (IHC) for pERK, pAKT, and pVEGFR-2. $B R A F$ mutation status was determined by DNA sequencing. ${ }^{16}$ Of the first 15 patients treated, five patients achieved a partial response, three had stable disease, two progressed, and three patients with stable disease withdrew due to toxicity. Target lesions decreased on average 31\%. Eight of 10 PET scans showed decreased activity at four weeks. IHC on tissue from two patients showed 50\% decrease in pERK (downstream of VEGFR2 and BRAF) and 30\% decrease in pAKT (downstream of VEGFR2). In tissue from a patient at 17 months on sorafenib, the decrease in pERK and pAKT appeared to be the same or reversed, suggesting compensatory changes in these pathways in resistant but stable disease. In conclusion, this study showed the early clinical and biologic activity of sorafenib in patients with $\mathrm{mPTC}$ and the targets of early suppression. Importantly, it also revealed compensatory changes in target molecules in cells resistant to therapy. These cells are the likely source of tumor resistance that has been seen to develop to other similar targeted agents. ${ }^{16}$ When patients with all types of thyroid cancer were treated with this agent, the results were equally impressive although the majority of cases had either follicular or papillary subtypes. Thirty patients were treated with a response rate of $23 \%$ and a stable disease rate of $53 \%$. The median progression-free survival was 79 weeks. ${ }^{6}$ Phase II studies are currently still enrolling patients with other subtypes of thyroid cancer using this agent.

Targeted therapy with agents similar to the ones mentioned above appear to be useful in metastatic thyroid cancer treatment. Axitinib is a multitargeted agent against the VEGF receptors 1,2 , and 3 and will be evaluated further in this paper.

\section{Outcomes achieved in clinical development}

Axitinib is one of a number of new targeted therapies being evaluated in the treatment of many cancers. It inhibits 
the VEGF receptors at subnanomolar concentrations and also inhibits PDGFR-beta and c-Kit in low nanomolar concentrations. In a phase I trial designed not only to find the maximum tolerated dose, but also to look at the effect of food on pharmacokinetics, the planned starting dose of $30 \mathrm{mg}$ twice daily was found to be too toxic. ${ }^{17}$ A dose de-escalation scheme determined the recommended dose for phase II trials to be $5 \mathrm{mg}$ twice daily. Not surprisingly, the effect of food was found to decrease both the rate and extent of absorption and there was a median increase of $49 \%$ in the plasma exposures of the fasted compared with fed state. In the presence of a proton pump inhibitor, rabeprazole, the rate but not the extent of absorption was decreased, and so the concurrent use of this group of medications was felt to be not clinically significant. Twelve-hour urinary collections obtained on days 1 and 29, demonstrated that less than 1\% of the administered dose appeared as unchanged drug in the urine regardless of dose, indicating that the majority of drug elimination was through systemic metabolism. The primary dose limiting toxicity was hypertension. This appeared to be dose-related and was controlled easily with antihypertensive medications. However, it was occasionally severe, and in the first cohort at a dose of $30 \mathrm{mg}$ before hypertension guidelines were established, two patients suffered seizures although they recovered without sequelae. In the 14 patients treated at the recommended phase II dose, six developed hypertension, but it was not dose-limiting and was controlled by standard antihypertensive medications. Interestingly, no shifts in renin, angiotensin II, or aldosterone were seen, suggesting that axitinib-induced hypertension is not mediated by this pathway. One patient who had thyroid cancer had a reduction of tumor burden, but did not meet the RECIST criteria for a response. ${ }^{17}$

Based on these encouraging data, a phase II trial was performed using axitinib given at a dose of $5 \mathrm{mg}$ twice daily in patients with all types of aggressive incurable thyroid cancer. ${ }^{5}$ Patients with hemoptysis and uncontrolled hypertension were excluded from this study. In total, 60 patients were enrolled, 30 with papillary, 15 with follicular, and 11 with medullary cancer. A partial response was noted in 18 patients giving an overall response rate of $30 \%$. Stable disease was reported in another $23(38 \%)$ and four patients $(7 \%)$ had progressive disease as their best response. With a median follow up of 16.6 months, the median progression-free survival is 18.1 months which correlates well with the other small molecule studies mentioned above. The results were even more impressive as most of the patients enrolled were men and had metastatic disease, both poor prognostic factors. The most common adverse effects were fatigue, diarrhea, nausea, anorexia, and hypertension. Of these, hypertension was the most common grade 3 side effect, occurring in $12 \%$ of patients.

The effect of axitinib on soluble proteins as exploratory pharmacodynamic markers was also studied. Treatment with axitinib led to a 2.8-fold increase in mean VEGF concentrations that generally reached a plateau by week 12 . In contrast, there was a $32 \%$ decrease in mean sVEGFR-2 concentrations, 35\% decrease in mean sVEGFR-3 concentrations, and $13 \%$ decrease in mean sKIT concentrations in blood by week 12 compared with baseline. The decrease in sVEGFR reached a plateau within the first 12 weeks of treatment and was sustained for the duration of therapy in most patients. Given the paucity of patients with progressive disease, it was not possible to make a definitive assessment of the potential correlation of changes in sVEGFR-2 or sVEGFR-3 with objective response. However, these data demonstrate the selectivity of axitinib for modulating soluble VEGFRs versus sKIT in patients with thyroid cancer.

Calcitonin and thyroglobulin were both measured in subsets of patients in this study. Calcitonin was measured in seven patients with medullary thyroid cancer: two patients with progressive disease, three patients with stable disease, and two patients with partial response. Patients with progressive disease did not have a change in calcitonin concentrations; those with partial response had a marked decrease in calcitonin concentrations and patients with stable disease had a slight decrease in calcitonin.

Thyroglobulin was measured in two patients with progressive disease, 11 patients with stable disease, and eight patients with partial response. The preliminary assessment demonstrates that most patients, regardless of their clinical response to therapy, had initial decreases in thyroglobulin. However, because of the small number of patients with progressive disease in this trial, no definitive conclusions could be made for either calcitonin or thyroglobulin with regard to their utility as potential biomarkers of response to axitinib. ${ }^{5}$

For the most resistant thyroid cancers, monotherapy with axitinib may not be sufficient to control the disease. Chemotherapy has been used with some success in earlier thyroid cancer studies and therefore a combination of axitinib chemotherapy may represent another possible treatment option. Although these combinations have not been tested in the setting of thyroid cancer, they have been evaluated in other diseases. In two phase I studies reported at the 2008 ESMO meeting, axitinib was combined with a variety of chemotherapy regimens including FOLFOX and FOLFIRI in 
gastrointestinal malignancies and taxane-, capecitabine-, or gemcitabine-based treatments in a variety of solid malignancies. In both studies, the pharmacokinetics were not affected by the addition of chemotherapy and the toxicities were not increased. ${ }^{18,19}$ More involved studies have also explored axitinib chemotherapy combinations with other new agents including a PDGFR inhibitor CP-868,596 combined with docetaxel and axitinib. Once again, the combination appeared safe, pharmacokinetic interactions were not observed, and antitumor effect was noted. ${ }^{20}$ Similar results were seen when axitinib was combined with FOLFOX and bevacizumab. ${ }^{21}$

In these days of exotic correlative markers, an intriguing retrospective analysis of the axitinib trials performed to date noted that patients who developed hypertension with a diastolic blood pressure of greater than $90 \mathrm{mmHg}$ had a better survival than those whose did not become hypertensive. This appeared to be true for all tumor types, and in the case of thyroid cancer, the difference was 21.9 months survival for the nonhypertensive group compared with overall survival not reached for the hypertensive group of responders. ${ }^{22}$

\section{Ongoing studies}

Axitinib is currently being studied in an ongoing phase II trial in patients with metastatic thyroid cancers which are refractory to or ineligible for radioiodine therapy (NCT00176748). Another trial is currently enrolling patients who have ${ }^{131}$ I-Refractory metastatic or unresectable locally-advanced papillary, follicular, or Hurthle-cell thyroid cancer who are also refractory to, or intolerant of, or have clinical contraindication to, doxorubicin treatment (NCT00389441). Doxorubicin remains the only US Food and Drug Administration (FDA)-approved treatment for iodine-refractory metastatic thyroid cancer and if this study meets its accrual expectations, it will be the largest study using the new small molecule inhibitors in this disease to date. ${ }^{23}$

\section{Resource utilization}

Thyroid cancers are rising in incidence faster than most other cancers, and therefore even though they remain a relatively uncommon disease today, in future their management could potentially become increasingly important to endocrinologists and medical oncologists. As the numbers of cancers increase, so do the numbers of patients who have aggressive refractory disease. Agents such as axitinib appear to have a toxicity profile that is much more favorable than the standard chemotherapy options that are currently available for use in this disease. Therefore, axitinib and similar compounds could potentially find themselves in increasing demand if the current trend of increasing numbers of thyroid cancer continues.

\section{Patient group/population}

Age and male gender appear to be the two main poor prognostic factors in patients with thyroid cancers and therefore this is probably the population who would gain the most benefit from aggressive treatment of this disease. Younger female patients can often have a long lifespan often in the presence of widely metastatic disease. However it is important to take each case individually and if the disease appears particularly aggressive, then axitinib should be considered as an option for treatment when it becomes available. Interestingly, axitinib may also have a place in the treatment of medullary and anaplastic thyroid cancers although the numbers of patients with these subtypes is too small to draw firm conclusions.

\section{Clinical potential}

Axitinib appears to be a very promising treatment for patients with aggressive iodine-refractory thyroid cancer. There are at least two other multitargeted kinase inhibitors which also have significant activity. It is difficult to compare these treatments as they have all only been tested in single arm phase II studies. They all appear to be well tolerated. Phase III studies will be needed to determine whether these agents are better than the current standard of care and if they should be approved for thyroid cancer treatment. Certainly the toxicity of these agents appears better than that of traditional chemotherapy, but many of the chemotherapy studies were performed before modern antiemetics and antibiotics were widely available.

There have been no quality of life studies with axitinib when used as a treatment for thyroid cancer. However, the QLQ-C30 health-related quality of life (HRQOL) questionnaire was incorporated into a study using axitinib in patients with renal cell cancer. ${ }^{24}$ The results were encouraging and appeared to show that there was an increase in survival with little or modest change from baseline levels in the majority of HRQOL domains and symptoms. The one exception was diarrhea, which appeared to get worse during treatment, although the average score did not change response categories to 'a little' until 120 weeks, 2.5 years after the start of treatment, and was still at that approximate level by 144 weeks. Thyroid cancer and renal cell cancer often share characteristics of a slowly progressive malignancy and therefore these results may be applicable when axitinib is used in the setting of thyroid cancer as well. However, this may be one of the biggest challenges for the widespread 
use of these new small molecule inhibitors, as their initial cost is likely to be extremely high and many patients are asymptomatic even with widespread metastatic disease. Any benefit in survival should also be matched by an improvement in quality of life, as otherwise the economic burden of the treatment is likely to be prohibitive. However, the emergence of axitinib and its fellow compounds as viable options for treating refractory thyroid cancers represents a remarkable advance in treating these hitherto resistant tumors.

\section{Acknowledgments}

The authors report no conflicts of interest in this work.

\section{References}

1. Jemal A, Siegel R, Ward E, et al. Cancer statistics 2008. CA Cancer J Clin. 2008;58:71-96.

2. Colonna M, Guizard AV, Schvartz C, et al. A time trend analysis of papillary and follicular cancers as a function of tumour size: A study of data from six cancer registries in France (1983-2000). Eur J Cancer. 2007;43:891-900.

3. Deshpande H, Gettinger S, Sosa J. Novel chemotherapy options for advanced thyroid tumors: small molecules offer great hope. Curr Opin Oncol. 2008;20:19-24.

4. Hu-Lowe D, Hallin M, Feeley R, et al. Characterization of potency and activity of the VEGF/PDGF receptor tyrosine kinase inhibitor AG013736 [abstract]. Proc Am Assoc Cancer Res. 2002;43:5357.

5. Cohen E, Rosen L, Vokes E, et al. Axitinib is an active treatment for all histologic subtypes of advanced thyroid cancer: results from a phase II study. J Clin Oncol. 2008;26(29):4708-4713.

6. Gupta-Abramson V, Troxel A, Nellore A, et al. Phase II trial of sorafenib in advanced thyroid cancer. J Clin Oncol. 2008;26(29):4714-4719.

7. Sprague B, Andersen S, Trentham-Dietz A. Thyroid cancer incidence and socioeconomic indicators of health care access. Cancer Causes Control. 2008;19:585-593.

8. Hodgson N, Button J, Solorzano C. Thyroid cancer: Is the incidence still increasing? Ann Surg Oncol. 2004;11:1093-1097.

9. Carling T, Udelsman R. Cancer of the endocrine system: Section 2 thyroid tumors. In: De Vita V, Hellman S, Rosenberg S, editors. Cancer principles and practice. 8th Edition. Philadelphia: Lippincott Williams and Wilkins; 2008. p. 1664-1682.
10. Sosa J and Udelsman R. Total thyroidectomy for differentiated thyroid cancer. J Surg Oncol. 2006;94:701-707.

11. Sherman S. Early clinical studies of novel therapies for thyroid cancers. Endocrinol Metab Clin N Am. 2008;37:511-524.

12. Durante C, Haddy N, Baudin E, et al. Long-term outcome of 444 patients with distant metastases from papillary and follicular thyroid carcinoma: benefits and limits of radioiodine therapy. $J$ Clin Endocrinol Metab. 2006;91:2892-2899.

13. Shimaoka K, Schoenfeld DA, DeWys WD, et al. A randomized trial of doxorubicin versus doxorubicin plus cisplatin in patients with advanced thyroid carcinoma. Cancer. 1985;56:2155-2160.

14. Gupta V, Pattaswamy K, Lassoued W, et al. Sorafenib targets BRAF and VEGFR in metastatic thyroid carcinoma [abstract]. Proc Am Soc Clin Oncol. 2007;25:6019.

15. Sherman S, Wirth L, Droz J-P, et al. Motesanib diphosphate in progressive differentiated thyroid cancer. N Engl J Med. 2008; 359:31-42.

16. Cohen Y, Xing M, Mambo E, et al. BRAF mutation in papillary thyroid carcinoma. J Natl Cancer Inst. 2003;95:625-627.

17. Rugo H, Herbst R, Liu G, et al. Phase I trial of the oral antiangiogenesis agent AG-013736 in patients with advanced solid tumors: pharmacokinetic and clinical results. J Clin Oncol. 2005;3:5474-5483.

18. Martin L, Kosloff M, Herbst R, et al. Phase I study of axitinib (AG-013736) in combination with chemotherapy in patients with advanced solid tumors [abstract]. Ann Oncol. 2008;19:472.

19. Robles R, Burgess R, Infante J, et al. Axitinib (AG-013736; AG) in combination with chemotherapy (CT): A phase 1 study in patients (PTS) with gastrointestinal tumors [abstract]. Ann Oncol. 2008;19:473.

20. Michael M, Vlahovic G, Khamly K, Pierce K, Guo F, Ozlanski. A Phase Ib study of CP-868,596, a PDGFR inhibitor, in combination with docetaxel (Doc) with or without AG-013736, a VEGF inhibitor [abstract]. Proc Am Soc Clin Oncol. 2008;26:3549.

21. Abhyankar V, Sharma S, Trowbridge R, et al. Axitinib (AG-013736) in combination with FOLFOX and bevacizumab (bev) in patients (pts) with advanced solid tumors: A phase 1 study [abstract]. Proc Am Soc Clin Oncol. 2008;26:4112.

22. Rini B, Schiller J, Fruehauf J, et al. Association of diastolic blood pressure $(\mathrm{dBP}) \geq 90 \mathrm{mmHg}$ with overall survival (OS) in patients treated with axitinib (AG- 013736) [abstract]. Proc Am Soc Clin Oncol. 2008;26:3543.

23. Anon. Clinical Trials with axitinib and thyroid cancer. 2008. Cited 2008 Oct. Available from: http//www.clinicaltrials.gov/

24. Trask P, Bushmakin A, Cappelleri J, Bycott P, Liau K, Kim S. Health-related quality of life during treatment for renal cell carcinoma: Results from a phase II study of axitinib. Acta Oncol. 2008;47:843-851.
Core Evidence

\section{Publish your work in this journal}

Core Evidence is an international, peer-reviewed open-access journal evaluating the evidence underlying the potential place in therapy of drugs throughout their development lifecycle from preclinical to postlaunch. The focus of each review is to evaluate the case for a new drug or class in outcome terms in specific indications and patient

\section{Dovepress}

groups. The manuscript management system is completely online and includes a very quick and fair peer-review system, which is all easy to use. Visit http://www.dovepress.com/testimonials.php to read real quotes from published authors. 